\title{
Emergency Thoracic Endovascular Aneurysm Repair in Acute Type B Dissection with Visceral Malperfusion
}

\author{
Giuseppe Petrilli, $\mathrm{MD}^{1 *}$, Giovanni Puppini, $\mathrm{MD}^{2}$, Daniele Calzaferri, $\mathrm{MD}^{1}$, Salvo Torre, $\mathrm{MD}^{1}$, \\ Antonella Bugana, $\mathrm{MD}^{1}$, Giuseppe Faggian, $\mathrm{MD}^{1}$ \\ ${ }^{1}$ Division of Cardiac Surgery, University of Verona, Verona, Italy; ${ }^{2}$ Department of Radiology, University of Verona, Verona, Italy
}

\begin{abstract}
A 46-year-old female patient was admitted to the emergency room with the sudden onset of abdominal pain, back pain, and paresthesia in the right leg. An emergent chest computed tomography (CT) showed an acute Type B aortic dissection. An emergency thoracic endovascular aneurysm repair (TEVAR) procedure was subsequently performed, for an evolving visceral malperfusion syndrome. We performed the procedure using an axillary approach because the small diameter of the true lumen precluded transfemoral endovascular access. This case illustrates that TEVAR permits the treatment of complicated acute Type B aortic dissection; specifically, technical variations can expand the applicability of endovascular procedures. $\quad$ Copyright $\odot 2013$ Science International Corp.
\end{abstract}

\section{Key Words}

Emergency TEVAR - Acute type B dissection - Visceral malperfusion

\section{Introduction}

Acute Type B aortic dissection (ATBAD) is a lifethreatening condition. Open chest surgical repair using a prosthetic graft has been a conventional treatment for ATBAD. During the past decade, thoracic endovascular aortic repair (TEVAR), which is considered a less invasive and potentially safer technique, has been increasingly used to treat this condition [1]

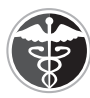

Fax +1 2037853346

E-Mail: aorta@scienceinternational.org

http://aorta.scienceinternational.org
(C) 2013 Aorta.

Published by Science International Corp.

ISSN 2325-4637

Accessible online at: http://aorta.scienceinternational.org
A 46-year-old female patient was admitted at our institution with ATBAD. We are reporting a clinically successful result from the TEVAR used for distal malperfusion syndrome that occurred as a consequence of the dissection. A particular technique was necessary. True lumen flow was immediately restored following stent graft deployment in the descending aorta, with subsequent resolution of the distal malperfusion syndrome.

\section{Clinical Case}

A 46-year-old female patient was admitted to the emergency room of our hospital with the sudden onset of abdominal pain, back pain, and right leg weakness. At the time of presentation, blood pressure, heart rate, and $\mathrm{O}_{2}$ saturation were 185/100 $\mathrm{mm} \mathrm{Hg}, 61$ beats/min, and $100 \%$, respectively. A cyanotic pulseless right lower extremity and diminished bowel sound were physically notable.

An emergent chest computed tomography (CT) was performed under the suspicion of an acute aortic dissection, which showed an acute Type B aortic dissection, starting just distal to the left subclavian artery and extending to the terminal aorta. The pressurized false lumen encroached on the central true lumen of the

${ }^{*}$ Corresponding author:

Giuseppe Petrilli, MD

Division of Cardiac Surgery

University of Verona

Piazzale Stefani 1

37100 Verona, Italy

Tel: +390458123307, Fax: +390458123308, E-Mail: gio.petrilli@gmail.com 


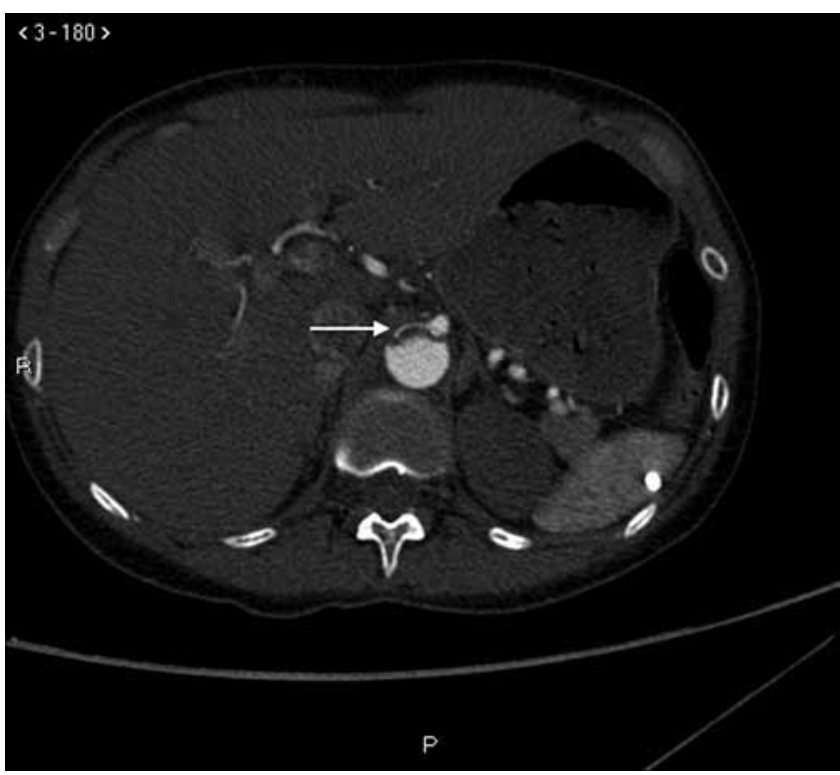

Figure 1. Pre-operative $C T$ scan. The white arrow indicates the virtual true lumen at the level of the celiac trunk.

abdominal aorta; the superior mesenteric artery was arising from the true lumen, the right renal artery was arising at the turn of the two lumens and the inferior left renal artery from the false lumen (Fig. 1); indeed, the true lumens of the superior left renal and inferior mesenteric artery were compressed by the false lumen.

An emergency TEVAR procedure was subsequently performed, for an evolving visceral malperfusion syndrome, to close the proximal entry site, relieve the aortic false lumen pressure, and reestablish central lumen flow.

The patient was transferred to an angiographic interventional room. The patient underwent general anesthesia, and a standard cut-down over the right femoral artery and percutaneous left axillary access were performed. We attempted to advance the guide wire into the iliac side branch from the right femoral access, but this maneuver failed because of the virtual dimensions of the true lumen. For this reason, a soft wire was advanced retrogradely via the axillary artery and then anterogradely down the aorta into the right iliac artery. Then, the soft wire was snared by a wireloop, which carried the soft wire in the femoral artery (Fig. 2). Using this approach, we were able to advance one tapered Relay NBS 30-26X150 (Bolton Medical Inc. Sunrise, Florida, USA and Barcelona, Spain) stent-graft from the right femoral access into the descending aorta through the true lumen. We positioned the graft from the origin of the left subclavian artery (partially

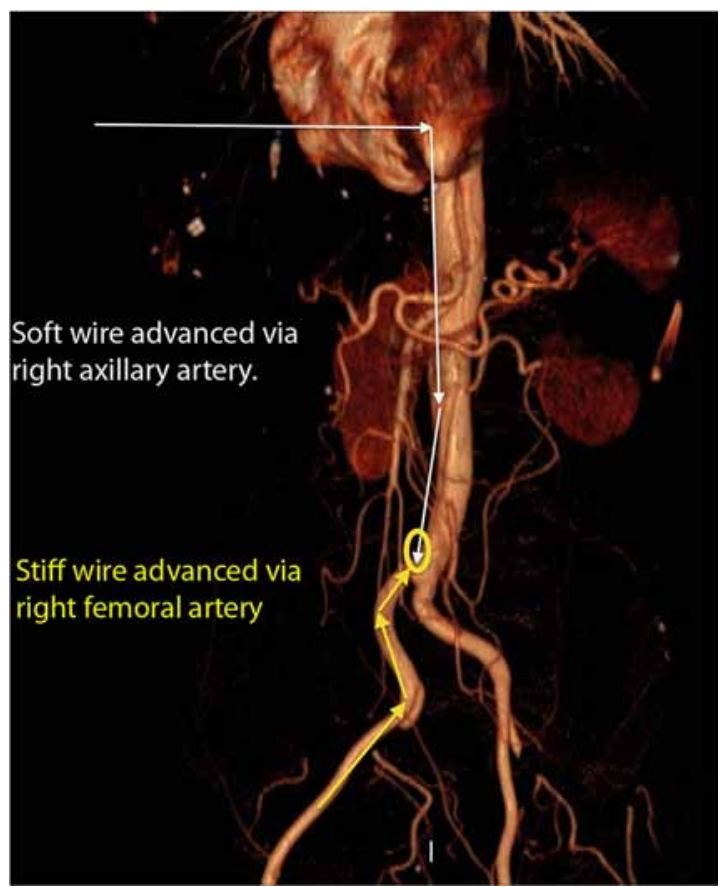

Figure 2. CT scan showing the directions in which the wires were passed.

covered) to the distal descending thoracic aorta. Immediate intraoperative postprocedural aortography showed restoration of central true lumen flow and enhanced flow to the visceral vessels.

Prior to discharge, a follow-up CT showed the TEVAR procedure was successful by indicating a good restoration of the central aortic true lumen (Fig. 3) and visceral artery patency (Fig. 4); particularly, the right renal artery was arising entirely from the true lumen and the left superior renal artery and inferior mesenteric artery were no longer compressed by the false lumen. Although the left inferior renal artery was still arising from the false lumen, the kidney showed excellent dye enhancement without the need for postoperative hemodialysis.

\section{Discussion}

TEVAR, initially developed for the treatment of degenerative aneurysms of the descending thoracic aorta, has been applied to the entire spectrum of descending thoracic aortic pathology in both the elective and emergent settings. According to Mitchell et al. [2], TEVAR could become the treatment of choice for acute surgical emergencies involving the descend- 


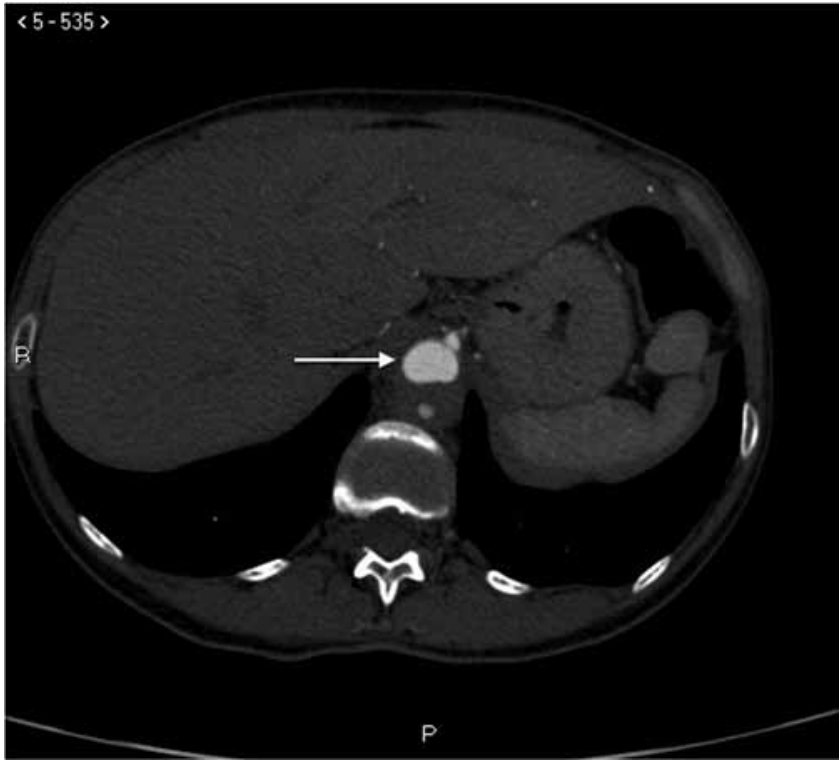

Figure 3. Postoperative CT scan. The white arrow indicates the virtual true lumen at the level of the celiac trunk.

ing thoracic aorta, including acute complicated Type B dissection. The role of TEVAR in the management of this pathology consists in covering the entry tear during the acute phase, inducing false lumen thrombosis, and possibly preventing late aneurysm formation. Indeed, as reported by Conrad et al. [3], TEVAR for acute complicated aortic dissection appears to promote early aortic remodeling, and nearly $90 \%$ of patients maintained at least partial false lumen thrombosis at 1 year.

Because TEVAR eliminates the basic elements of surgical intervention (i.e. thoracotomy, left heart bypass, aortic cross-clamping, and deep hypothermic circulatory arrest), this new therapeutic modality is believed to lower the risks of morbidity and mortality in descending thoracic aortic aneurysm, traumatic aortic rupture, and type B aortic dissection. Related to this, in contrast to high mortality of open surgery for thoracic aortic catastrophes, including traumatic aortic injury, excellent short-term outcomes following TEVAR have recently been reported $[4,5]$.

Many individuals who are candidates for TEVAR are found to have iliac artery anatomy and/or pathology that may preclude a transfemoral endovascular surgi-

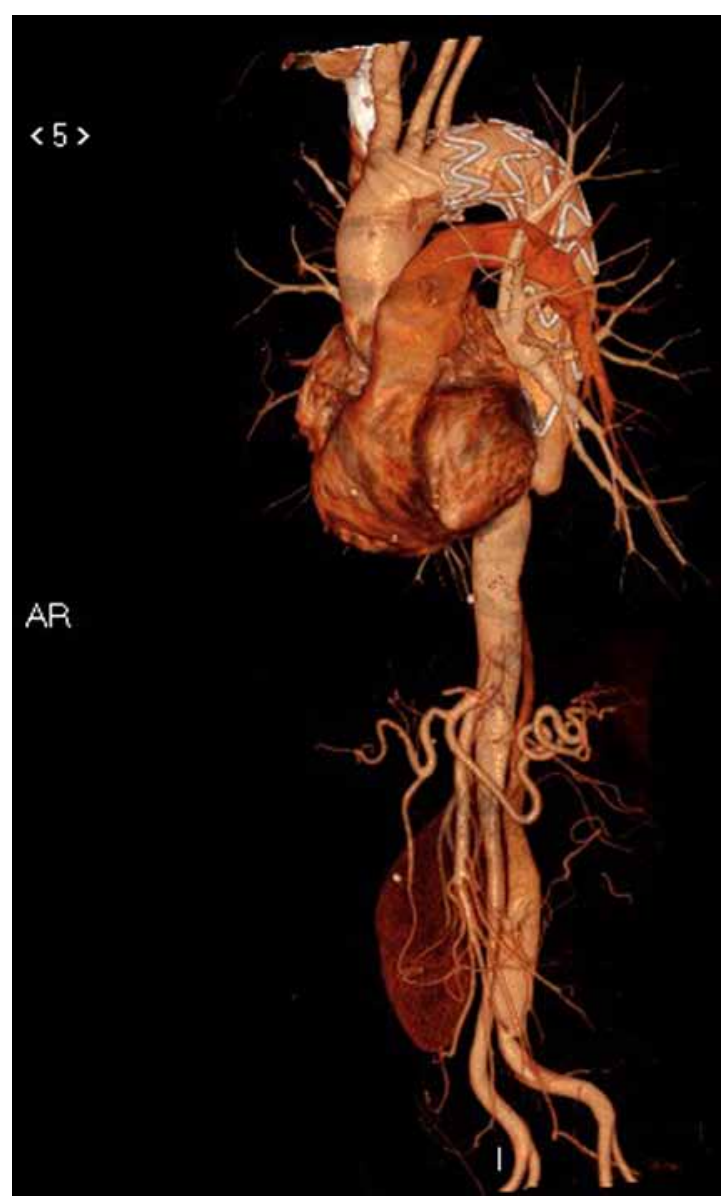

Figure 4. At 30 days follow-up, CT showing the correct placement of stent graft.

cal approach. Imaginative alternate surgical access through more proximal arteries may be necessary and fruitful. Indeed, with inadequate true lumen iliac artery dimension, an axillary endovascular approach permits this technique for safe implantation of a stent graft in the descending aorta-as illustrated in this case.

In conclusion, TEVAR permits the treatment of complicated acute descending aortic dissection, with efficiency and security. Importantly, technical tips and tricks, such as the technique described in this report, can expand the applicability of endovascular technology to descending aortic dissection.

\section{Comment on this Article or Ask a Question}

\section{References}

1. Zhang H, Wang ZW, Zhou Z, Hu XP, Wu HB, Guo Y. Endovascular stent-graft placement or open surgery for the treatment of acute type $B$ aortic dissection: a meta-analysis. Ann Vasc
Surg. 2012;26:454-461. 10.1016/j.avsg.2011. 09.004 
2. Mitchell ME, Rushton FW Jr, Boland AB, Byrd TC, Baldwin ZK. Emergency procedures on the descending thoracic aorta in the endovascular era. J Vasc Surg. 2011;54:1298-1302. 10.1016/j.jvs.2011.05.010

3. Conrad MF, Crawford RS, Kwolek CJ, Brewster DC, Brady TJ, Cambria RP. Aortic remodeling after endovascular repair of acute complicated type B aortic dissection. J Vasc Surg. 2009;50:510-517. 10.1016/j.jvs.2009. 04.038
4. Azizzadeh A, Charlton-Ouw KM, Chen Z, Rahler $M H$, Estrera $A L, A m e r ~ H$, et al. An outcome analysis of endovascular versus open repair of blunt traumatic aortic injuries. J Vasc Surg 2013; 57:108-114. 10.1016/j.jvs.2012.05.110

5. Carmona AF, Redondo AD, Pareja JC, Maldonado CP. Endovascular treatment of descending thoracic aortic rupture: mid- to long-term results in a single-centre registry. J Cardiovasc Med (Hagerstown). 2012;13:266-268. 10.2459/ JCM.0b013e3283515b61
Cite this article as: Petrilli G, Puppini G, Calzaferri D, Torre S, Bugana A, Faggian G. Emergency Thoracic Endovascular Aneurysm Repair in Acute Type B Dissection with Visceral Malperfusion. Aorta 2013;1(3):206-209. DOl: http://dx.doi.org/ 10.12945/j.aorta.2013.13-009

\section{EDITOR'S QUESTIONS}

1. Loss of the left renal artery is a serious shortcoming of this procedure? Please comment. Are you considering late revascularization of this artery to preserve renal function? Is this ischemic kidney causing refractory renovascular hypertension?

In this case the left kidney was perfused by two arteries, superior and inferior. At presentation just the upper part of the kidney was completely ischemic because the corresponding artery was compressed by the false lumen. The left inferior renal artery was perfused by the false lumen, while the right renal artery was from both lumens. After the procedure the right kidney was completely reperfused, while the left was mostly reperfused; however, no impairment of renal function was evident. Refractory renovascular hypertension was seen initially, but disappeared after the procedure.

2. Many experts would argue against your uniform characterization of descending aortic surgical procedures as "high mortality." Please comment, keeping in mind contemporary surgical results.
Descending aortic pathologies such as Type B dissection, descending thoracic aortic aneurysm, penetrating ulcers (PAU), and acute traumatic aortic injuries still represent a potentially life-threatening situation and a formidable risk for the patient and the cardiac surgeon. Conventional resection and graft replacement has been the treatment of choice, but is still associated with high morbidity and mortality despite improved surgical techniques. Meanwhile, thoracic endovascular aneurysm repair (TEVAR) has evolved into a safe and effective therapy for all these different pathologies. Nevertheless, patients with acute Type $B$ aortic dissections and acute traumatic aortic lesions seem to benefit the most from TEVAR.

3. Many Type B dissections produce a very smallappearing, crescentic true lumen, but, paradoxically, this works very well, with excellent distal pulses and perfusion. Do you consider such a radiographic appearance an indication for TEVAR therapy?

No. I think that in such cases there is no indication for TEVAR, but the patient should be followed closely for the high possibility of developing complications that may require treatment by TEVAR. 